\title{
Meta
}

Journal des traducteurs

Translators' Journal

\section{Delisle, Jean et Judith Woodworth (dir) (1995) : Les traducteurs dans l'histoire, Ottawa, Presses de l'Université d'Ottawa et éditions UNESCO, 348 p.}

\section{Christian Balliu}

Volume 41, numéro 3, septembre 1996

URI : https://id.erudit.org/iderudit/001963ar

DOI : https://doi.org/10.7202/001963ar

Aller au sommaire du numéro

Éditeur(s)

Les Presses de l'Université de Montréal

ISSN

0026-0452 (imprimé)

1492-1421 (numérique)

Découvrir la revue

Citer ce compte rendu

Balliu, C. (1996). Compte rendu de [Delisle, Jean et Judith Woodworth (dir) (1995) : Les traducteurs dans l'histoire, Ottawa, Presses de l'Université d'Ottawa et éditions UNESCO, 348 p.] Meta, 41(3), 473-475.

https://doi.org/10.7202/001963ar d'utilisation que vous pouvez consulter en ligne.

https://apropos.erudit.org/fr/usagers/politique-dutilisation/ 
DELISLE, Jean et Judith WOODSWORTH (dir.) (1995) : Les traducteurs dans l'histoire, Ottawa, Presses de l'Université d'Ottawa et éditions UNESCO, 348 p.

Ce projet ambitieux est loin d'être neuf puisque le IVe Congrès de la F.I.T., tenu à Dubrovnik en 1963, insistait sur la nécessité d'écrire une histoire universelle de la traduction. György Radó répétera ce besoin lors du Congrès de Lahti de 1966, qui voit par ailleurs la création du Comité pour l'histoire de la traduction. L'ampleur d'un tel ouvrage, qui se doit de couvrir plusieurs centaines de langues en privilégiant une dimension culturelle diachronique, ne pouvait se concevoir sans la collaboration de plusieurs équipes de chercheurs, dirigée chacune par un rédacteur principal. C'est cette option qui fut retenue lors du Congrès de Belgrade de 1990, où la coordination fut prise en charge par Jean Delisle et Judith Woodsworth. De même, on s'est accordé sur une analyse thématique et sélective, soucieuse d'éviter un eurocentrisme trop prononcé. Cette option est respectée dans l'ensemble, même sì l'Europe reste un passage obligé puisqu'elle fournit actuellement le plus de recherches dans le domaine et qu'elle fut, du moins avant le XVIe siècle, le pôle principal de l'activité traduisante.

Le livre est sans conteste un ouvrage de vulgarisation, destiné aux lecteurs du monde entier, ce qui ne peut étonner lorsqu'on connaît la vocation universaliste de la F.I.T. Le traducteur y est mis en exergue à travers les neuf thèmes suivants : les traducteurs inventeurs d'alphabets, bâtisseurs de langues nationales, artisans de littératures nationales, diffuseurs des connaissances, acteurs sur la scène du pouvoir, propagateurs des religions, importateurs de valeurs culturelles et rédacteurs de dictionnaires; enfin, les interprètes, témoins privilégiés de l'histoire.

Ce découpage est évidemment arbitraire et adopté pour la commodité de la composition; il reste entendu que les personnages qui défilent sous nos yeux apparaissent sous différentes bannières, ce qui ne fait que confirmer que le savoir et sa transmission sont en prise directe avec le pouvoir et le contexte socioculturel de l'époque.

Si les traducteurs sont replacés dans leur cadre historique, ce dernier n'est pas toujours appréhendé dans sa juste dimension. Le cours événementiel ne traduit pas constamment les textures sociales et politiques qui ont nourri le travail du traducteur. J'en veux pour preuve les quelques exemples qui suivent.

L'œuvre de Mesrop Machtots ne peut se comprendre sans souligner la lutte d'influence qu'a dû mener l'empire byzantin contre la force du mazdéisme iranien en Arménie. Cyrille et Méthode font quant à eux partie d'une longue lignée d'écoles de traducteurs, nées très tôt chez les Slaves du Sud et de l'Est, et dont l'archétype est à rechercher chez les chroniqueurs de l'époque. Outre les missions khazare, sarrasine et du Vardar, la mission morave de 863 traduit une fois encore le lien indéfectible qui unit les traducteurs et les interprètes à la diplomatie, tant il est vrai que la Pax romana va de pair avec la Pax christiana. Ce lien est remarquable aussi chez les princes d'Éléphantine ou plus tard dans l'école des Enfants de Langues à partir de 1669. Par ailleurs, la lutte intra-byzantine pour 
admettre la traduction des Saintes Écritures en langues vulgaires est indissociable de l'activité de Cyrille. Ne disait-il pas que «apprendre sans alphabet et sans livres revient à noter une conversation sur l'eaul»?

Les figures de proue cachent parfois une forêt de traducteurs qui en sont les héritiers et les continuateurs. C'est ainsi que toute une littérature de traduction s'épanouira sous la houlette du basileus Syméon, dans sa nouvelle capitale de Preslav, aux confins de la Bulgarie orientale. C'est notamment de la sorte que Jean Chrysostome, dont Syméon admirait les œuvres, fut traduit dans les littératures slaves naissantes. L'homilétique alimentera d'ailleurs l'activité traduisante à Byzance.

En outre, la différentiation entre glagolitique et cyrillique dès le $X^{e}$ siècle se cristallisera précisément autour de la rivalité entre Ohrid, restée fidèle au glagolitique malgré saint Clément, et Preslav, qui verra l'essor du cyrillique. La traduction ne naît pas ex nihilo, pour reprendre l'expression de Cary, mais épouse les mouvements d'une époque dont elle est un témoin privilégié.

La traduction biblique, domaine délicat s'il en est, trouve logiquement sa place dans le chapitre consacré aux «traducteurs, propagateurs de religions». Le passage concernant saint Jérôme me semble à certains endroits peu précis et aurait mérité un autre développement insistant davantage sur l'influence particulière de la littérature profane dans sa formation. D'autre part, la réduction de ses conceptions traduisantes à la lettre à Pammachius, héritage de Valery Larbaud qui y voyait «la porte monumentale par laquelle on accède à l'œuvre de traducteur de Jérôme ${ }^{2}$ », fait l'impasse sur les options contradictoires qui ont jalonné sa vie de traducteur des Saintes Ecritures. Malheureusement, les Hexaples d'Origène ne sont pas mentionnés et, à ce propos, Symmaque et Théodotion ne font l'objet d'aucun traitement, alors qu'Aquila trouve fort justement sa place dans le livre.

L'oubli de certains personnages est sans doute la rançon de ce genre d'ouvrages. Il est vrai qu'il faut aller à l'essentiel, chose ardue s'il en est en histoire de la traduction, vu la jeunesse de la discipline. Les traducteurs de l'ombre peuvent parfois jouer un rôle prépondérant, mais le hasard des recherches ne les a pas toujours placés sous les feux de la rampe. Peut-être leur importance sera-t-elle découverte plus tard et seront-ils alors mis en lumière. À cet égard, il est un peu curieux de trouver sous l'étiquette «traducteurs, bâtisseurs de langues nationales» un Claude de Seyssel, pourtant spécialiste de la traduction indirecte et dont le travail se caractérisait par une volonté délibérée de latiniser le français. Plusieurs traducteurs de l'ombre, qui ont œuvré à son service, sont passés sous silence: Laurent Valla ou Jean Lascaris ${ }^{3}$ auraient sans aucun doute mérité d'être repris pour leur aptitude à traduire en langues classiques. Lascaris fut à Paris le maître de Guillaume Budé et rapporta de ses voyages en Grèce nombre de manuscrits anciens, bénéficiant notamment du mécénat de Laurent le Magnifique, grand protecteur des savants et féru d'imprimerie. Comme ce fut le cas à d'autres époques - citons par exemple Hunayn Ibn Ishaq -, l'office de traducteur englobait d'autres tâches tout aussi importantes, comme la collecte de sources fiables ou la diffusion des productions. Mais il est vrai que l'immense mouvement de traductions caractéristique de l'époque postbyzantine doit encore être étudié et apprécié à sa juste valeur.

En matière d'interprétation, l'accent est mis à juste titre sur l'époque contemporaine, même s'il me semble que la distribution chronologique et géographique du chapitre aurait pu être plus heureuse et ne pas économiser l'évolution de la profession. En outre, il est étonnant d'apprendre que l'absence de mention des interprètes dans les annales historiques serait imputable au fait qu'ils «étaient souvent des femmes, des esclaves ou des membres d'une caste inférieure» (p. 244). Ce n'était certainement pas le cas en Égypte ancienne ni à Bagdad, encore moins au XVII siècle français. L'école des Enfants de 
Langues, que Mounin considérait comme le début de la traduction diplomatique, ne reçoit pas le développement qu'elle mérite si l'on considère l'étendue de son activité. Elle est d'ailleurs à inscrire dans le vaste mouvement de «turqueries» qui fleurira désormais en France et qui culminera avec les Lettres persanes. A l'opposé de ce qu'écrivent les auteurs du chapitre (p. 272, note 20), l'idée de former des interprètes uniquement français ne perdura que jusqu'en 1700 , année où l'échec des capucins de Péra fut consommé. L'âge d'or de l'école des Enfants de Langues, sise au Collège Louis-le-Grand et inféodée à la Compagnie, durera de 1721 à 1762 et sera marqué par un cursus que l'on pourrait qualifier d'hypermoderne.

Enfin, le chapitre consacré aux traducteurs rédacteurs de dictionnaires fait souvent l'impasse sur la situation politique, culturelle et linguistique de l'époque envisagée. Les dictionnaires reflètent inéluctablement les préoccupations non seulement langagières, mais aussi et surtout «techniques» de chaque société à un moment particulier de son évolution.

Toutefois, on peut dire que, de manière générale, l'ouvrage est très vivant et illustre des épisodes trop souvent méconnus en histoire de la traduction, notamment en ce qui concerne l'Afrique et l'Asie. Il s'agit de ce qu'on pourrait appeler une petite histoire illustrée qui, loin de se vouloir exhaustive, constitue une introduction de choix dans le domaine fascinant de l'histoire de la traduction. Le traitement scientifique des sujets est à souligner, même si l'on regrettera certaines erreurs comme la mention de codex incas (p. 159, note 12) ou encore des positions parfois trop catégoriques, comme les dates de naissance et de mort de saint Jérôme $(331-420)$ ou la «notoriété durable» de Jacques Amyot (p. 52), contredite sans ambages par le discours à l'Académie de Bachet de Méziriac en 1635.

La bibliographie de base donnée à la fin de chaque chapitre comprend les ouvrages essentiels qui permettront au lecteur d'approfondir le domaine particulier qui l'intéresse. Un index des noms cités autorise une recherche plus ciblée, sans recourir à la structure thématique.

Christian BALLIU

Institut supérieur de traducteurs et interprètes, Bruxelles, Belgique

Notes

1. Cité par D. MARKOV (1978) : «Les Slaves - culture et histoire», Courrier de l'Unesco, août-sept. 1978, p. 4.

2. Sous l' invocation de saint Jérôme, Gallimard, 1946, p. 15.

3. La famille constantinopolitaine des Lascaris, qui donna d'ailleurs à l'empire byzantin plusieurs empereurs, a fourni à l'Occident des traducteurs, dont Constantin Lascaris, après la débâcle de 1453. Leur origine princière et byzantine leur assurait une connaissance remarquable du grec et du latin. 